\title{
A Common Low Back Pain Hiding Tuberculoussacro-Iliitis
}

\author{
Hiba Ben Ayed ${ }^{1,2^{*}}$ and S Miladi, \\ ${ }^{1}$ Rheumatology Department, Mongi Slim Hospital, Tunisia \\ ${ }^{2}$ Faculty of Medicine of Tunis, Tunis El Manar University, Tunisia
}

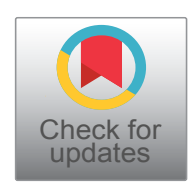

*Corresponding author: Hiba Ben Ayed, Rheumatology Department, Mongi Slim Hospital, La Marsa, Tunisia; Faculty of Medicine of Tunis, Tunis El Manar University, Tunis, Tunisia

\section{Case Report}

Isolated tuberculosis of the sacrum is rarely reported in the literature, mostly limited to sporadic cases, with a frequency estimated at for approximately 0.3 to $0.5 \%$ of all tuberculosis cases [1-3]. Its presenting symptoms are non-specific, with a predominance of vague backpain [4].

Herein, we report the case of a 61-year-old healthy female who visited our outpatient unit complaining about a history of low back pain lasting for 2 years. Her symptoms predominated on the right side with no radicular irradiation. Her low back pain increased while efforts and decreased at rest with no night awakeness. Her pain did not relieve under paracetamol. Otherwise, she did not complain about arthralgia, cutaneous eruption, ocular manifestations or respiratory symptoms. Besides, she did not report constitutional symptoms such as fever, weight loss, asthenia or anorexia. Her BCG vaccination had been performed during childhood. She reported being in contact with a family member who had lymph node tuberculosis.

Physical examination revealed a temperature of 37.5 ${ }^{\circ} \mathrm{C}$. A $3 \mathrm{~cm}$ Schober test was measured and straight-leg raise was negative. The range of motion of the hips was normal. Evidence of sacroiliac disease was found, with Patrick-Fabere's test positive for the left hip pain and upon pressure on the left iliac wings and over the left sacroiliac joint was painful. We noted a non tender swelling measuring $3 \times 3 \mathrm{~cm}$ situated in the right hip region with a normal overlying skin. There were no sensory or motor changes in either lower limb.

Laboratory tests showed inflammation (erythrocyte sedimentation rate, $40 \mathrm{~mm} / \mathrm{h}$; C-reactive protein, 19 $\mathrm{mg} / \mathrm{l})$. Blood cell counts were normal. A tuberculin skin test was positive, with an in duration of $15 \mathrm{~mm}$.

A plain radiograph of the pelvis showed widening of the left sacroiliac joint space with erosions of the subchondral bone (Figure 1). Magnetic resonance imaging (MRI) confirmed the radiographic findings of left sacro-iliitis and showed a collection in the left sacro-iliac joint and in the left iliopsoas muscle with an extension to the sacral spinal canal (Figure 2). A fistula was born from an opening inside the sacral spinal canal, along the first sacral nerve root, to an outside opening in the left gluteal area, resulting in an abscess measuring $3.8 \times 14.4 \mathrm{~cm}$. Chest radiograph was normal.

Considering these aspects, the most likely diagnosis was tuberculoussacro-iliitis. Percutaneous drainage of the abscess under CT guidance was performed. Smears of the aspirated material did not reveal acid fast bacilli and the culture on Lowenstein-Jensen medium was also negative but PCR performed on the specimen was positive for Mycobacterium tuberculosis.

The patient was started on four-drug antituberculous therapy. She experienced significant improvement soon after the initiation of the treatment. Currently after three months of treatment (two months of intensive phase followed by five months of daily isoniazid and rifampicin), she only complains of a minimal discomfort on her back pain with no relapse of the swelling.

Sacroiliac joint tuberculosis is an uncommon and elusive clinical diagnosis [2]. An early diagnosis requires a high index of clinical suspicion.

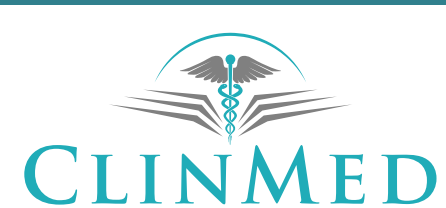

INTERNATIONAL LIBRARY

Citation: Ayed HB, Miladi S (2021) A Common Low Back Pain Hiding Tuberculoussacro-lliitis. Int J Radiol Imaging Technol 7:080. doi.org/10.23937/2572-3235.1510080

Accepted: November 11, 2021; Published: November 13, 2021

Copyright: (C) 2021 Ayed HB, et al. This is an open-access article distributed under the terms of the Creative Commons Attribution License, which permits unrestricted use, distribution, and reproduction in any medium, provided the original author and source are credited 


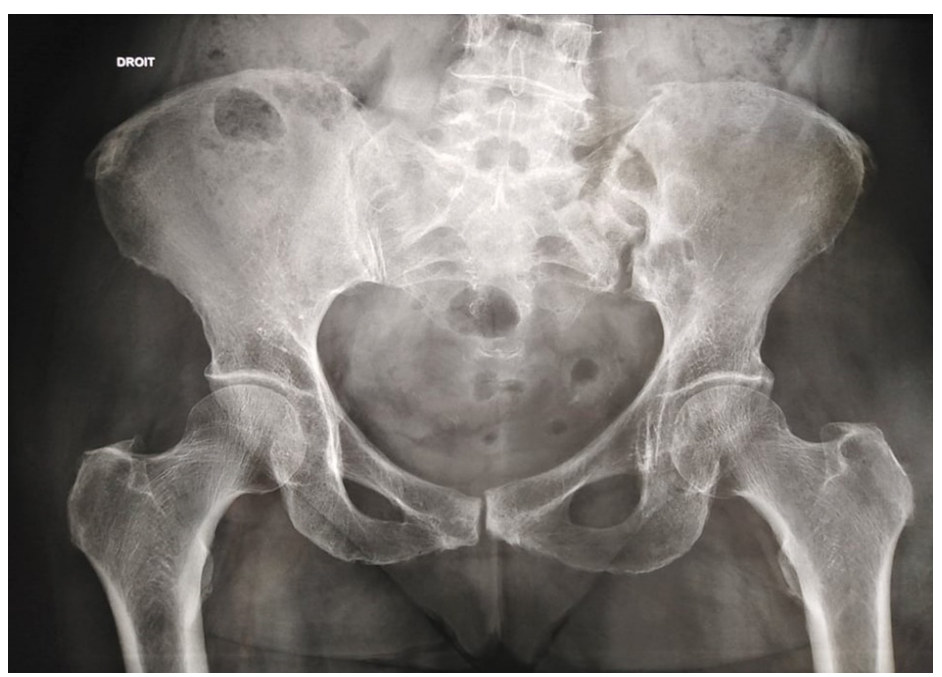

Figure 1: Plain radiograph of the pelvis showed widening of the left sacroiliac joint space with erosions of the subchondral bone.

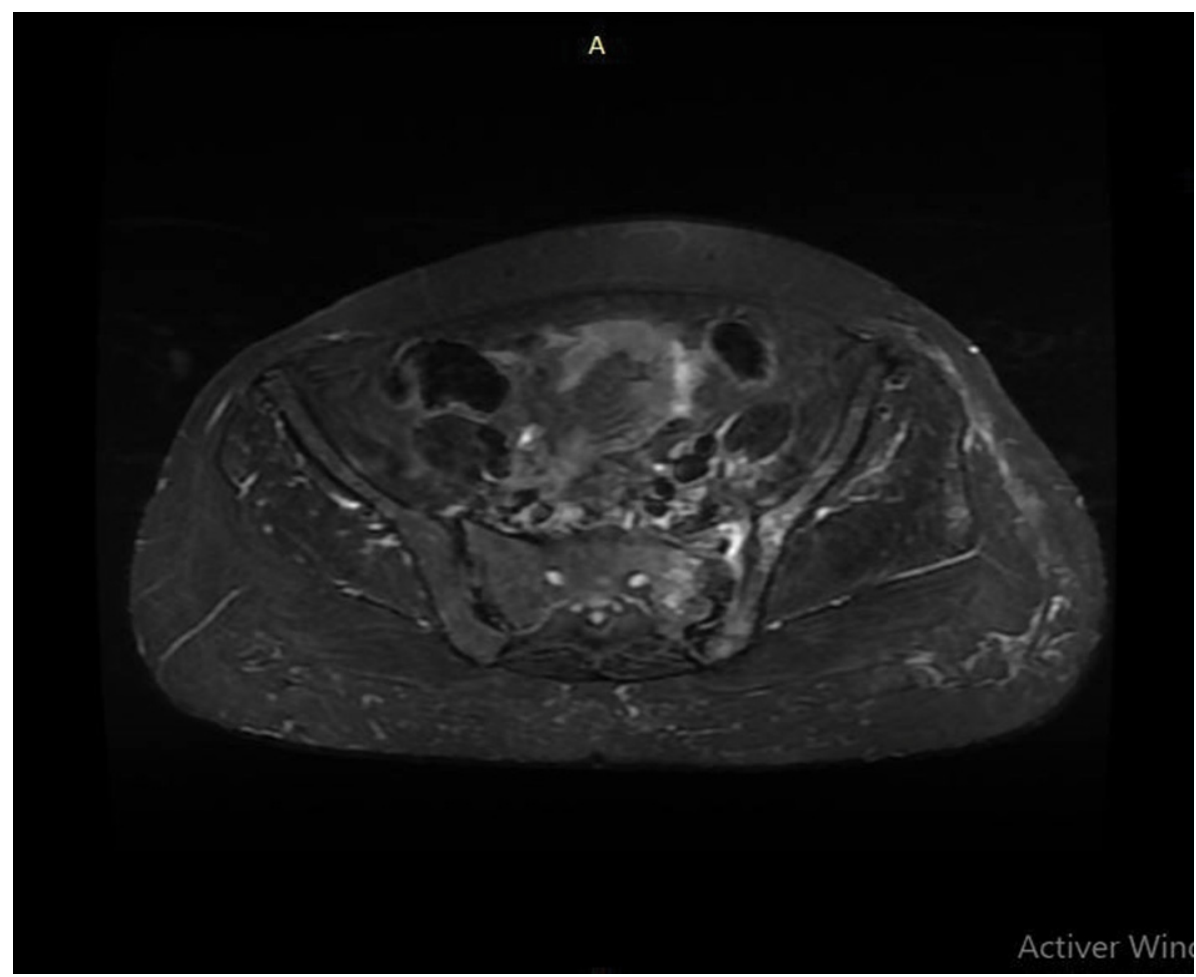

Figure 2: Magnetic resonance imaging (MRI) of the pelvis showing a left sacro-iliitis and a collection in the left sacro-iliac joint (arrow).

\section{Conflict of Interest}

\section{None.}

\section{Funding Disclosure}

No funding received.

\section{Author Contributions}

Author 1 and author 2: Collected the data and wrote the paper; Author 3 and author 4: Contributed in data collection; Others: Provided grammatical revisions to manuscript.

\section{Acknowledgements}

None.

\section{References}

1. Osman AA, Govender S (1995) Septic sacroiliitis. Clin Ortho Relat Res 313: 214-219.

2. Ramlakan RJS, Govender S (2007) Sacroiliac joint tuberculosis. Int Orthop 31: 121-124.

3. Soholt ST (1951) Tuberculosis of the sacroiliac joint. J Bone Joint Surg Am 33: 119-130.

4. Chandradevan R, Takeda H, Lim T, Patel N (2020) Mycobacterium tuberculosis concealed by enterococcal sacroiliitis. IDCases 21: e00858. 